\title{
REFERENCES
}

1. E. A. Coddington and N. Levinson, Theory of ordinary differential equations, New York, McGraw-Hill Co., 1955.

2. J. Cronin, Note to Poincarê's perturbation method, Duke Math. J. vol. 26 (1959) pp. 251-262.

3. - Poincare's perturbation method and topological degree, to appear in Contributions to Nonlinear Differential Equations, vol. 5, Princeton University Press.

4. - Branch points of solutions of equations in Banach space. II, Trans. Amer. Math. Soc. vol. 76 (1954) pp. 207-222.

5. A. Sard, The measure of the critical values of differentiable maps, Bull. Amer. Math. Soc. vol. 48 (1942) pp. 883-890.

Polytechnic Institute of Brooklyn

\section{QUOTIENT GROUPS OF REDUCED ABELIAN GROUPS}

\section{ELBERT A. WALKER}

Let $G$ be a reduced torsion $p$-group. (In this paper, group will mean Abelian group.) Let $B$ be a basic subgroup of $G$. It is well known that $|B| \wedge_{0} \geqq|G|$, where $|S|$ denotes the cardinal of the set $S$. Fuchs gives a proof of this in $[1$, p. 102], and attributes it to Kulikov. This has turned out to be a very useful fact, and the purpose of this short note is to generalize it. Now, as is generally known, every torsion group $G$ has a basic subgroup $B$; that is, a pure subgroup $B$ that is a direct sum of cyclic groups, and such that $G / B$ is divisible. To obtain such a $B$, simply take $B_{p}$ to be a basic subgroup of the $p$-component of $G$, and let $B=\sum_{p} \oplus B_{p}$. It is easy to see that in this more general situation $|B| \aleph_{0} \geqq|G|$ still holds, and in fact follows from the corresponding statement for $p$-groups. The generalization we will prove is the following

THEOREM. Let $G$ be a reduced torsion group, and let $H$ be a subgroup of $G$ such that $G / H$ is divisible. Then $|H| \aleph_{0} \geqq|G|$.

Proof. Our proof uses some homological results of Harrison in [2]. Notice that we may assume that $|H|<|G|$, that $G$ is infinite, and hence that $|G / H|=|G|$. Let $Q$ and $Z$ denote the additive group of rationals and integers, respectively. From the exact sequence

$$
0 \rightarrow H \rightarrow G \rightarrow G / H \rightarrow 0,
$$

we get the exact sequence

Received by the editors March 17, 1960. 


$$
0 \rightarrow \operatorname{Hom}(Q / Z, G / H) \rightarrow \operatorname{Ext}(Q / Z, H) \rightarrow \operatorname{Ext}(Q / Z, G) \rightarrow 0,
$$

since $H$ and $G$ are reduced, and $Q / Z$ and $G / H$ are divisible. If $H$ is of bounded order, then $\operatorname{Ext}(Q / Z, H) \cong H$. (See [2].) However, $\operatorname{Hom}(Q / Z, G / H)$ is torsion-free $[2$, p. 371], so that $H$ must be of unbounded order, and in particular $|H| \geqq \boldsymbol{N}_{0}$. Therefore we may assume $|G|>2^{N_{0}}$. Hence $G / H$ is the direct sum of $|G|$ copies of $Z\left(p^{\infty}\right)^{\prime}$ s, for various primes $p$. Now for a particular $Z\left(p^{\infty}\right),\left|\operatorname{Hom}\left(Q / Z, Z\left(p^{\infty}\right)\right)\right|$ $=2 \aleph_{0}$, being the $p$-adic integers. Thus $|\operatorname{Hom}(Q / Z, G / H)| \geqq 2 \aleph_{0}|G|$ $=|G|$, and from the exact sequence above we see that $\operatorname{Ext}(Q / Z, H)$ has a subgroup of cardinal $|G|$. But $\operatorname{Ext}(Q / Z, H)$ is a quotient group of a group of functions of two variables of $Q / Z$ into $H$ (see [2, p. 368]) and hence $|\operatorname{Ext}(Q / Z, H)| \leqq|H| \aleph^{\aleph_{0}}$. Therefore $|H| \aleph_{0} \geqq|G|$.

We conclude with the following remarks. First, if $H$ is a basic subgroup, we have obviously shown $|H| \aleph_{0} \geqq|G|$. Secondly, suppose $H$ is of bounded order. Write $G / H=D \oplus R$, where $D$ is divisible and $R$ is reduced. $\operatorname{Hom}(Q / Z, G / H)=\operatorname{Hom}(Q / Z, D \oplus R) \cong \operatorname{Hom}(Q / Z, D)$ $\oplus \operatorname{Hom}(Q / Z, R) . \operatorname{Hom}(Q / Z, D)$ is torsion-free and is isomorphic to a subgroup of $\operatorname{Ext}(Q / Z, H) \cong H$. Hence $D=0$ since $D$ is torsion, and we see that if $H$ is a subgroup of $G$ of bounded order, then $G / H$ is reduced. Finally, we easily see from the proof of our theorem above that if $G / H=D \oplus R$, with $D$ divisible, $R$ reduced, and $|D|=|G|$, then $|H| \aleph_{0}$ $\geqq|G|$.

\section{REFERENCES}

1. L. Fuchs, Abelian groups, Budapest, Publishing House of the Hungarian Academy of Sciences, 1958.

2. D. K. Harrison, Infinite Abelian groups and homological methods, Ann. of Math. vol. 69 (1959) pp. 366-391.

New Mexico State University 